\title{
The Development of GPS Navigation Systems in Civil Aircraft.
}

\begin{abstract}
The industry of civil aviation is developing in a fast manner to occupy the increasing needs for a fast, safe and comfortable transportation. To achieve these needs, some effective programs, plans, and systems designs are required. The current reliable aircraft navigation and landing systems have relatively a low level of accuracy especially in aircraft landing stage. The best landing category could be achieved requires a runway visual range up to 50 meters and automatic landing system is not in operation yet all over the world. Aircraft navigates using Radar, Radio and Satellite navigation systems. The Federal Aviation Administration (FAA) plans to replace legacy navigation systems with satellite based navigation technology. Currently, the Global Positioning System (GPS) and its augmentations is the main satellite navigation system used all over the world for air navigation applications. The development of aircraft navigation has been performed with the enhancement of GPS augmentation systems. In this paper, aircraft navigation systems and techniques will be presented in this paper to evaluate the integrity and reliability of each system, and to make a comparison among these systems according to accuracy, integrity and availability.
\end{abstract}

Keyword: Aircraft Navigation; Augmentation system; DGPS; GPS; LAAS; WAAS; Landing Systems 